\title{
HISTORIA Y FILOSOFÍA DE LAS CIENCIAS EN LA EDUCACIÓN POLIMODAL: PROPUESTA PARA SU INCORPORACIÓN AL AULA
}

\author{
History and Philosophy of Science in Science Curricula: a \\ proposal for its incorporation in Science Classes
}

\author{
Verónica Guridi ${ }^{1}$ \\ Irene Arriassecq ${ }^{2}$
}

\begin{abstract}
Resumo: Durante los últimos años se viene enfatizando la necesidad de la incorporación de la Historia y Filosofía de las Ciencias (HFC, en adelante) a los currículos de Ciencias. Mucho se ha avanzado respecto de la definición de los marcos teóricos que debieran orientar la incorporación de la HFC en la Enseñanza de las Ciencias, pero sin embargo son pocos los trabajos que muestran propuestas concretas de incorporación de la HFC en las clases de Ciencias. Este trabajo intenta realizar un aporte en el sentido antes mencionado, presentando una propuesta para el tratamiento de un núcleo temático específico correspondiente al primer año Polimodal (orientación Humanidades y Ciencias Sociales) en el espacio curricular Físico - Química. El núcleo temático seleccionado corresponde al estudio de la evolución de los modelos atómicos (hasta el modelo atómico de Bohr).
\end{abstract}

Unitermos: Historia y Filosofía de la Ciencia - Enseñanza de Ciencias - Enseñanza Media - Modelos Atómicos.

Abstract: In the last few years the necessity of including History and Philosophy of Science (HFC, hereafter) in Science Curricula has been emphasised. There have been important advances in theoretical frameworks, which should orient the incorporation of HFC into Science classes. However, there are only a few works that include proposals that show some ways of making this concrete. This work attempts to make a contribution in that direction, presenting a proposal for the treatment of a specific thematic nucleus of the curricular area "Physics-Chemistry". The proposal is designed for the first year of High School Education (with orientation in Humanities and Social Sciences). The selected content corresponds to the study of the evolution of atomic models (up to the model of Bohr).

Keywords: History and Philosophy of Science - Science Teaching - High School Education - Atomic Models

\section{Introducción}

En los últimos años se ha enfatizado sobre la incorporación de la HFC a los currículos de Ciencias. Particularmente, hay trabajos que señalan la necesidad de la incorporación de HFC a los profesorados de Ciencias (Sandoval y Cudmani, 1993; Guridi et al, 1997). Otro grupo de trabajos de índole más general tiende a caracterizar al conocimiento científico y derivar de allí implicaciones para la enseñanza de las Ciencias o a caracterizar la aproximación actual de la HFC para la enseñanza (Matthews, 1994; Mora Penagos, 1997). Un tercer grupo de trabajos de índole teórica se centra en una revisión crítica desde la Historia de las Ciencias sobre temas específicos de Física, por ejemplo, electromagnetismo y mecánica (Velazco, 1999; Córdoba y Gramajo, 1999; Furió y Guisasola, 1993, por citar algunos). También hay trabajos

\footnotetext{
${ }^{1}$ Alumna regular del Programa de Pos-Graduación en Educación (opción Enseñanza de Ciencias y Matemática), Facultad de Educación, Universidad de San Pablo, Brasil. Con apoyo del CNPq. (e-mail: veguridi@yahoo.com.ar)

${ }^{2}$ Profesora Adjunta, Departamento de Formación Docente, Facultad de Ciencias Exactas, Universidad Nacional del Centro de la Provincia de Buenos Aires, Argentina.

Alumna regular del Programa Internacional de Doctorado en Enseñanza de las Ciencias, Universidad de Burgos (España) y UFRGS (Brasil). (e-mail:irenearr@exa.unicen.edu.ar)
} 
que analizan la incorporación de la HFC en los textos de Física posteriores a la actual Reforma Educativa. En esa línea se encuentran trabajos como el de Arriassecq y Stipcich (2000), que analizan críticamente la incorporación de la HFC en nueve textos de Física post-reforma educativa. Entre las conclusiones señaladas por las autoras, caben destacar dos de ellas: la incorporación de la HFC en los textos escolares obedece más a una "cuestión de moda" que al marco teórico consensuado en HFC y, además, que la mayor parte de las propuestas de incorporación de HFC quedan en la teoría, con lo cual se hace necesario incorporar propuestas concretas de tratamiento a nivel áulico.

Algunos libros de texto proponen un tratamiento consistente con los resultados de investigaciones acerca de la HFC, pero estos textos no son de uso frecuente entre los docentes de Ciencias (Holton y Brush, 1976; Hecht, 1987). Uno de los textos recientemente editados, que ya está circulando entre los docentes de Física y áreas afines (no incorporado en la muestra analizada por Arriassecq y Stipcich (2000)), propone un tratamiento interesante, también consistente con los presupuestos teóricos defendidos en el área de investigación en Enseñanza de las Ciencias. Es el caso del texto de Candás et al (2000) (este texto ha sido utilizado para la elaboración de algunas de las actividades presentadas en este trabajo).

Una última línea de trabajos asume el enfoque CTS, que defiende la incorporación de la HFC en la educación científica como parte de la formación de una conciencia ciudadana en los estudiantes (Aduriz Bravo et al, 2002).

Se hace necesario, por tanto, incorporar los aportes de las investigaciones acerca de la HFC en propuestas de aula, aunque se sigan consensuando criterios teóricos. Podría afirmarse con cierto rigor que ya existe un hábeas de conocimientos teóricos que han sido elaborados por la comunidad de investigadores, debidamente discutidos y fundamentados.

Recientemente, hay trabajos que realizaron aportes en el sentido de incorporar la Historia de la Ciencia por medio del teatro (De Oliveira, 2004) o que intentaron incorporar la Historia de la Ciencia al análisis de datos de investigación referidos al trabajo experimental de estudiantes en el laboratorio didáctico (Dias, 2004).

Este trabajo, que va en una dirección convergente, presenta una propuesta concreta de incorporación de la HFC a nivel áulico, con ejemplos de actividades para el trabajo con los estudiantes, recuperando el cuerpo de conocimientos propios de la HFC desde la investigación educativa en Ciencias.

\section{Marco teórico que orienta la incorporaciós de la HCF en la educación en ciencias}

\section{I.1. Contribuciones a la HFC desde la investigación educativa en Ciencias}

En la introducción a este trabajo se comentaba que existen varios trabajos que realizan aportes sobre la posible incorporación de la HFC en la educación científica Algunos trabajos se han centrado en la incorporación de la HFC a los currículos de los profesorados universitarios de Física y afines. Guridi et al (1997) enfatizan la necesidad de incorporar una visión contextualizada de la HFC para no formar en los estudiantes una imagen distorsionada sobre la naturaleza del conocimiento científico, sus formas de producción y de validación. En ese trabajo se señala que, desde la currícula de los profesorados, debiera fomentarse en los estudiantes una visión de Ciencia basada en los siguientes principios generales, extraídos de Guridi et al (1997), p. 260: a) La observación está mediada por las percepciones sensoriales que pueden ser falibles; b) la observación depende de la teoría y en algunos casos ésta es anterior a 
las observaciones; c) los conceptos y las teorías no surgen por generalización de observaciones sino que son creaciones que surgen a través de la abstracción; d) los conceptos y las teorías evolucionan; pueden modificarse e incluso rechazarse; e) no es adecuado promover métodos de aprendizaje por descubrimiento que generan una imagen inadecuada de la Ciencias, presentando a la inducción como descripción del método científico. Esto es, presentar a la Ciencia como una actividad neutral, que no posee relación alguna con cuestiones sociales, históricas o económicas y a los científicos como "personas todopoderosas capaces de determinar la verdad sobre el universo".

El supuesto subyacente a estos trabajos es que si los futuros docentes toman conciencia de estos criterios, pueden transferirlos a sus clases futuras de Ciencias. Se pretende generar una corriente de cambio que impregne todos los niveles educativos.

Otros trabajos, como el de Mora Penagos (1997) afirman que: “... la Ciencia, su enseñanza, su aprendizaje y su epistemología pueden ser tratados como un sistema complejo en donde la conexión entre la reflexión sobre la construcción de las Ciencias y la reflexión sobre su enseñanza y aprendizaje supone, en mayor o menor medida una reconstrucción de la actividad intelectual de los científicos (el subrayado no corresponde al autor) que justifica su aplicación en la enseñanza de las Ciencias en muchos aspectos dentro de los que se destacan: a) La comprensión de los mecanismos de producción de conocimientos de las Ciencias y de su filosofía (...); b) Desmitificar las Ciencias, ofreciéndole al alumno una imagen de las ciencias más ajustada a la realidad que manejan los científicos, permitiéndoles participar en diferentes procesos relacionados con la construcción del conocimiento científico (Mora Penagos, 1997, pp. 135-136).

Gil Pérez, (1986, 1994) realiza observaciones similares a las anteriores. Mora Penagos (op. cit., pp. 138-139) distingue dos imágenes mitificadas de la Ciencia: una, producto de un empirismo radical (la negrita no corresponde al autor), que sobrevalora la observación libre de teorías, y la segunda, producto de una exagerada y rigurosa racionalidad proyectada por la comunidad científica (la negrita no corresponde al autor), que fue forjada por los avances de la Física durante los últimos cien años.

Esta visión de la Ciencia ha estado (y aún lo sigue estando) presente tanto en los escritos pedagógicos como en la mayor parte de los textos escolares de Ciencias que circulaban tanto en el nivel medio como universitario básico. Inclusive, aún sigue estando presente en algunos textos escolares como fue mostrado en el trabajo ya citado de Arriassecq y Stipcich (2000).

Una última línea de trabajos asume que dentro de la Didáctica de las Ciencias el conocimiento de determinados tópicos de Filosofía de la Ciencia, tales como evidencia, método y explicación, pueden ayudar a los ciudadanos a tomar decisiones más críticas acerca de importantes temas científicos y tecnológicos en las sociedades democráticas. Así, existe un consenso en que es necesario que el ciudadano del siglo XXI no sólo conozca ciencia sino que también sepa cómo es creada y validada, cómo se desenvuelve a través de la Historia y cómo se relaciona con el medio social y cultural. Es el llamado enfoque CTS, que defiende la incorporación de la HFC en la educación científica como parte de la formación de una conciencia ciudadana en los estudiantes (Aduriz Bravo et al, 2002).

Podría decirse que la incorporación de la HFC en la Educación en Ciencias ha obedecido fundamentalmente a la necesidad de erradicar esta imagen estereotipada de la Ciencia y su metodología de trabajo, para dar lugar a aproximaciones más realistas acerca del quehacer científico pretendiendo, con ello, generar también actitudes más positivas de los alumnos hacia la Ciencia y posibilitar el desarrollo, en esos alumnos, de un pensamiento crítico necesario para actuar en sociedad. 


\section{2. Aportes desde la Historia de las Ciencias}

Desde la Historia de las Ciencias, autores que se encuentran cercanos a la educación científica como G. Boido, por ejemplo, han trazado líneas directrices para la incorporación de la Historia de las Ciencias en la Educación Científica. El marco teórico que define este autor se centra en torno de las dos grandes corrientes en Historia de la Ciencia que se han seguido desde los orígenes de la disciplina. Boido (1996, pp. 41 - 42) distingue entre la historiografía "whig" (o anacrónica) y la historiografia "antiwhig" (o diacrónica). La primera visión concibe a la Historia de la Ciencia como un relato de teorías, una visión "progresista" de la Historia de la Ciencia. Esta corriente en Historia de la Ciencia, como el mismo Boido comenta, fue producto de una influencia del positivismo de comienzos de siglo. La cuestión central que inquieta al historiador "whig" es el proceso que llevó a la construcción de la Ciencia y, por ello, ha de tomar de cada época en estudio los elementos que considere relevantes para la formación del espíritu científico subsiguiente. Es decir: se consideran sólo los aportes que derivaron en "éxitos" y no los fracasos para la Ciencia posterior. Por ejemplo, la teoría corpuscular de la luz de Newton se considera un "desliz" del científico y por ello no merece ser estudiada.

Sin embargo, algunos historiadores como Butterfield (críticos de la historiografía whig) señalan que esta corriente proporciona una visión distorsionada de la Historia de la Ciencia. El defecto más grande del que adolece esta visión es el anacronismo. Consecuentemente, estos autores proponen una visión diacrónica o "antiwhig" de la Historia de la Ciencia., que consiste en que el historiador se sitúe como un "viajero del tiempo", es decir, estudiar los acontecimientos históricos del pasado en virtud de las creencias, teorías, métodos y otros aspectos importantes de la propia época en la cual fueron gestadas las ideas.

El historiador diacrónico intenta ponerse lentes a través de los cuales mira la Historia. Desde esta perspectiva, se brinda una imagen más realista acerca de la Historia, en la cual no se ignoran los obstáculos y los errores propios del trabajo científico. De esta manera, la visión acerca de la Ciencia que se transmite también resulta más realista en el sentido de contemplar con el mismo peso los "aciertos" y los "fallos".

Boido concluye que estas dos visiones no deben tomarse como excluyentes sino como complementarias y, dependiendo del problema en cuestión, se utilizan elementos de una o de otra.

Muchas dificultades con la Física surgen debido a una incorrecta visión acerca de la naturaleza de la Ciencia. Los estudiantes no dimensionan el verdadero valor de la Física como Ciencia generadora de modelos y teorías para interpretar la realidad. Muchas veces, la Historia de la misma Ciencia Física "rebasa" las capacidades evolutivas de los estudiantes. Vale decir, no podemos pretender que un estudiante de nivel Polimodal reproduzca los experimentos de Rutherford, Einstein u otros. Sería una ingenuidad considerar que el estudiante está preparado para ello y no siempre es posible por cuestiones obvias de infraestructura. Alternativamente, puede resultar adecuado "reconstruir la Historia" en términos de Lakatos (2001). Esto es: ¿por qué no presentar documentos históricos de lectura a nuestros estudiantes y que ellos puedan "reconstruir" el proceso de elaboración de modelos, leyes y teorías? Esto último ha sido propuesto en varios trabajos de investigación educativa en Ciencias referidos a la incorporación de la HFC en la educación científica.

A modo de síntesis, podemos decir que del marco teórico anteriormente descripto se desprenden, entonces, los siguientes criterios que orientarían la incorporación crítica de la HFC en la Educación Científica:

a) Presentar ejemplos históricos en los cuales se visualice el proceso de elaboración de modelos, leyes y teorías, que incorporen no sólo aspectos conceptuales propios de 
la Física sino también creencias, actitudes, controversias y otros aspectos "extracientíficos" presentes en todo proceso de investigación científica.

b) Realizar una "reconstrucción racional" de las Ciencias de modo tal que, ya que a los estudiantes no les es ni psicológicamente ni temporalmente plausible reproducir un determinado proceso de investigación, puedan ser capaces de reconstruir la Ciencia, tomando conocimiento de ese proceso a través de la lectura y análisis de fragmentos de historia de la Ciencia. Esto supone que el estudiante se coloque en una perspectiva de "historiador novato" frente al proceso que analice.

Retomando esa línea, la propuesta que se presenta intenta "reconstruir" la evolución histórica acerca de los modelos atómicos, desde el descubrimiento de las primeras partículas subatómicas hasta el modelo atómico de Bohr (si bien sería muy interesante, no se puede abarcar la evolución hasta el modelo atómico actual por una cuestión de recorte necesario a los fines de este trabajo).

\section{Propuesta de trabajo para el primer año polimodal}

En la modalidad de Humanidades y Ciencias Sociales el énfasis está puesto en la vinculación de la Física con otras Ciencias, en particular con la Química. Por otra parte, la tendencia es la de estudiar la Física en un nivel conceptual, sin demasiados desarrollos matemáticos que obstaculicen la comprensión física de los conceptos trabajados. Siempre, y específicamente en esa modalidad, resulta conveniente presentar a la Física contextualizada socialmente, viéndola como una Ciencia que recibe aportes de diversa índole, siendo particularmente influenciada por factores sociales, políticos, religiosos, etc.

Por otra parte, desde los lineamientos curriculares para la Educación Polimodal, se distinguen cinco categorías de contenidos procedimentales presentados en los Contenidos Básicos Comunes para la Educación Polimodal (MEC, 1998), a saber:

- La formulación de problemas y explicaciones provisorias;

- La selección, recolección y registro organizado de la información;

- La interpretación de la información;

- El diseño de investigaciones;

- La comunicación de la información.

La incorporación de la HFC en la Educación Polimodal es una herramienta transversal a las cinco categorías anteriormente mencionadas.

\section{1. Contenidos a abordar}

\section{Físico - Quimica}

Modelos atómicos. Aportes del estudio de la electricidad. Descubrimiento del electrón. Descubrimiento del protón. Modelo del "budín” de J. J. Thompson. Aportes desde la radioactividad. El experimento de Rutherford. La luz y la estructura atómica. Las ondas electromagnéticas como herramientas para el estudio del átomo. El modelo atómico de Bohr (se señalan sólo los contenidos más relevantes). 


\section{$H F C$}

Descubrimiento o hallazgo. Experiencia. Hipótesis, hipótesis rivales, leyes científicas. Deducción, explicación, predicción, corroboración, refutación. Refutacionismo ingenuo. Realismo e instrumentalismo. Metodología del trabajo científico. Etapas (definición del problema, elaboración de hipótesis, diseño de experimentos, puesta a prueba de esos experimentos, interpretación de los resultados y formulación de conclusiones).

Influencia de los conocimientos previos en la formulación de una hipótesis. Evolución histórica de las ideas. Influencia de las creencias extracientíficas en las hipótesis elaboradas por los científicos. Discusión diacronismo - anacronismo en la Historia de la Ciencia. Aportes de diferentes científicos. Ciencia como cuerpo de conocimientos en continua evolución.

\section{2. Recursos para el trabajo en clase}

De acuerdo con el marco teórico explicitado, no todos los textos de Física que circulan en el nivel Polimodal son consistentes con el tratamiento que desde esta propuesta se pretende fomentar. Para esta propuesta se ha seleccionado la siguiente bibliografía, que, como fue comentado antes, resulta consistente con el marco teórico referido a la HFC en la Educación Científica, aunque, lógicamente, se trata de una sugerencia, que no impide que otros materiales puedan ser incorporados:

- Hecht, E. (1987) Física en Perspectiva. Editorial Addison Wesley Iberoamericana, cap. 10 (completo) y cap. 11 (pp. $299-316$ );

- Candás, A. et al (2000) Física y Química. Propiedades y transformaciones de la energía. Serie Estrada Polimodal. Editorial Estrada., pp. $30-34\left(^{*}\right)$.

$\left(^{*}\right)$ Este último puede darse como material complementario al anterior.

\section{3. Conocimientos previos de los estudiantes}

Se supone que los estudiantes ya poseen los siguientes conocimientos previos (que también corresponden al primer año Polimodal):

Las ideas de la materia en la antigüedad. El estudio de las reacciones químicas. La teoría del flogisto. Las primeras leyes cuantitativas de la Química: Boyle, Lavoisier. Teoría atómica de Dalton. Los aportes de Avogadro.

\section{4. Modalidad de trabajo en clase y actividades de los estudiantes}

Los estudiantes se dividirán en grupos y se entregará el material bibliográfico señalado con anterioridad. Se prevé que el núcleo temático ocupe al menos diez horas reloj (actualmente equivalente a un módulo en la educación Polimodal). Para la carga horaria de Físico-Química esto representa unas tres semanas de trabajo.

Durante los encuentros, los estudiantes irán leyendo y discutiendo en pequeños grupos el material de trabajo. Para orientar la discusión, se entregará a los mismos una guía de actividades que luego serán discutidas. La guía de actividades se muestra en el apéndice.

\section{5. Modalidad de evaluación}

Para que los estudiantes exterioricen los significados atribuidos por ellos a cada experiencia histórica y a cada modelo y que, además, el docente pueda observar si se contextualizó 
adecuadamente un determinado aporte histórico, se propone que los estudiantes realicen un mapa conceptual acerca del ejemplo histórico trabajado en clase. Junto con el mapa, cada grupo de estudiantes entregará una breve explicación del mismo. Esto exige un gran poder de síntesis pero, al mismo tiempo, exige no olvidar la contextualización de las ideas.

En instancias posteriores, se podrán exponer estos mapas y el docente puede implementar otras alternativas de evaluación.

\section{Comentarios finales}

¿Cuál es la novedad que introduce esta propuesta? Seguramente que algunos docentes ya han realizado tratamientos similares a este trabajo en algunas de sus clases de Física. Sin embargo, muchas veces la incorporación de la HFC se realiza sin previa reflexión, esto es: se realiza sin que se reconozca que se están incorporando numerosos contenidos de la HFC en las aulas.

Hacer conscientes los contenidos que se están abordando permite incorporarlos en forma funcional en las clases y no sólo cuando el tema "se preste para esto". Se puede pensar en una planificación en la cual la HFC y la Física interaccionen continuamente. No debería olvidarse que el propósito fundamental de la incorporación de la HFC en la educación científica tiene que ver, entre otras cosas, con el de presentar a los estudiantes una visión contextualizada de la Ciencia Física y de erradicar visiones "simplistas" y utópicas acerca de la metodología científica.

Por último, cabe destacar que la afectividad también se ve involucrada desde este nuevo enfoque. Justamente uno de los argumentos a favor de la incorporación de la HFC en las clases de Ciencias tiene que ver con la generación de actitudes positivas frente a la Ciencia. Uno de los autores de este trabajo ha puesto en práctica algunas de las actividades que se proponen aquí y es interesante mencionar que los estudiantes discuten fervorosamente entre ellos sobre determinadas cuestiones. Por ejemplo, cuando se estaba comentando que Rutherford planteó a Geiger y Marsden el desafío de encontrar partículas a que se desviaran a grandes ángulos, algunos estudiantes defendían la postura de que Rutherford ya conocía de antemano que no podían encontrarlas porque creía en el modelo de Thompson, mientras que otros sostenían que Rutherford no tenía confianza en ese modelo y por eso encomendó a sus colaboradores esa tarea, sospechando ya la existencia del núcleo. Interesantes líneas de trabajo en el aula pueden derivarse de lo anteriormente comentado.

En definitiva, son varias las alternativas que se pueden presentar. Inclusive, el lector puede encontrar otras no señaladas aquí. De las particularidades de cada grupo y del énfasis que el docente quiera poner en un determinado aspecto y no en otros, surgirá una variante a la propuesta. Inclusive, de las actividades que aquí se proponen, se pueden realizar sólo algunas de ellas, dependiendo del tiempo y recursos disponibles.

\section{Referencias}

ADURIZ BRAVO, A. et al. Three aspects when teaching the philosophy of science to science teachers. Disponível em: http://www1.phys.uu.nl/esera2003/programme/pdf/012S.pdf. Acesso em: 19 de março de 2004.

ARRIASSECQ, I.; STIPCICH, S. La visión de Ciencia en los textos de Física de nivel básico. In: V Simposio de Investigadores en Educación en Física, 2000, Santa Fe. Atas do V SIEF. CD-ROM. Santa Fé, Universidad Nacional del Litoral, v. único, [s. p.], 2000.

BOIDO, G. Noticias del Planeta Tierra. Buenos Aires: Editorial AZ, 1990. 
CANDÁS, A. et al. Física y Química. Propiedades y transformaciones de la materia y de la energía. Buenos Aires: Editorial Estrada, 2000.

CÓRDOBA, D.; GRAMAJO, C. La localización de la energía electrostática: un cambio ontológico no percibido. In: XI Reunión Nacional de Educación en Física, 1999, Mendoza. Atas REF XI. Mendoza, Universidad Nacional de Cuyo, v. único, p. 135-142, 1999.

DIAS, V. S. Michael Faraday: subsídios para metodologia de trabalho experimental. 2004. $157 \mathrm{f}$. Dissertação (Mestrado)- ENSCIENC - Interunidades em Ensino de Ciências da Universidade de São Paulo, São Paulo, 2004.

FURIÓ, C.; GUISASOLA, J. ¿Puede ayudar la historia de la ciencia a entender por qué los estudiantes no comprenden los conceptos de carga y potencial eléctrico? Revista Española de Física, Madrid, v. 7, n. 3, p. 46-50, 1993.

GIL-PÉREZ, D. La metodología científica y la enseñanza de las ciencias. Unas relaciones controvertidas. Enseñanza de las Ciencias, Barcelona, v. 4, n. 2, p. 111-121, 1986.

GIL-PÉREZ, D. Contribución de la historia y filosofía de las ciencias al desarrollo de un modelo de enseñanza/ aprendizaje por investigación. Enseñanza de las Ciencias, Barcelona, v. 11, n. 2, p. 197-212, 1994.

GURIDI, V.; ARRIASSECQ, I.; STIPCICH, S. Una propuesta de trabajo para el dictado de Historia y Epistemología de las Ciencias en la formación de profesores. In: VI Conferencia Interamericana sobre Educación en Física, 1997, Córdoba. Atas da VI Conferencia Interamericana sobre Educación en Física. Córdoba: Facultad de Matemática, Astronomía y Física, v. único, p. 259-264, 1997.

HECHT, E. Física en perspectiva. Delaware: Addison-Wesley, 1987.

HOLTON, G.; BRUSH, S. Introducción a los conceptos y teorías de las ciencias físicas. Barcelona: Editorial Reverté, 1976.

LAKATOS, I. Historia de la ciencia y sus reconstrucciones racionales. Madrid: Editorial Tecnos, 2001.

MATTHEWS, M. Science teaching: the role of history and philosophy of science. New York: Routledge, 1994.

MINISTERIO DE EDUCACIÓN. Contenidos básicos comunes para la Educación Polimodal. Buenos Aires: [s.n.], 1998.

MORA PENAGOS, W. M. Naturaleza del conocimiento científico e implicaciones didácticas. Revista Educación y Pedagogía, Medellín, v. 9, n.18, p. 131-144, 1997.

OLIVEIRA, N. R. de. A presença do teatro no ensino de física. 2004. 173 f. Dissertação (Mestrado)- ENSCIENC - Interunidades em Ensino de Ciências da Universidade de São Paulo, São Paulo, 2004.

SANDOVAL, J.; CUDMANI, L. Epistemología e historia de la física en la formación de los profesores de física. Revista Brasileira de Ensino de Física, São Paulo, v. 15, n. 1-4, p. 100-109, 1993. 
VELAZCO, S. Contribuciones de la historia de la física a la enseñanza del electromagnetismo. In: XI Reunión Nacional de Educación en Física, 1999, Mendoza. Atas REF XI. Mendoza, Universidad Nacional de Cuyo, v. único, p. 143-151,1999.

\section{Apéndice}

Guía de actividades (a la derecha, en negrita, aparecen los contenidos de HFC abordados con cada una de las actividades; hay otros contenidos trabajados con cada una de ellas pero se han señalado aquellos más relevantes para la resolución de la actividad en cuestión)

\section{Material bibliográfico:}

Hecht, E. (1987) Física en Perspectiva. Editorial Addison Wesley Iberoamericana, cap. 10 (completo) y cap. 11 (pp. $299-316)$

Candás, A. et al (2000) Física y Química. Propiedades y transformaciones de la energía. Serie Estrada Polimodal. Editorial Estrada., pp. 30 - 42.

\section{Actividades:}

Mediante la lectura del material bibliográfico, discutan con tus compañeros y elaboren las siguientes cuestiones:

1 - Organizando el trabajo... Para estudiar de manera ordenada la historia de la electricidad desde sus orígenes hasta el surgimiento de los primeros modelos atómicos, realicen el siguiente cuadro: (se completaron dos filas a modo de ejemplo)

\begin{tabular}{|c|c|c|c|c|}
\hline Año & Personaje & $\begin{array}{l}\text { Contribuiciones } \\
\text { (aportes) }\left({ }^{*}\right)\end{array}$ & Experiencias realizadas & Hallazgos \\
\hline & Gilbert & & & \\
\hline $\begin{array}{l}\text { Aprox. } \\
1650\end{array}$ & Von Guericke & $\begin{array}{l}\text { Construyó el primer gener- } \\
\text { ador electrostático }\end{array}$ & $\begin{array}{l}\text { Experiencias con diferentes materiales capaces de } \\
\text { ser electrizados }\end{array}$ & Electricidad a gran escala \\
\hline \multirow[t]{11}{*}{1700} & Du Fay & $\begin{array}{c}\text { Estudió las } \\
\text { interacciones } \\
\text { eléctricas }\end{array}$ & $\begin{array}{c}\text { Experiencias con esferas de azufre, experiencias con } \\
\text { pendulos electricos }\end{array}$ & $\begin{array}{l}\text { Descubre dos tipos de } \\
\text { electricidad }\end{array}$ \\
\hline & $\begin{array}{c}\text { Bernouilli } \\
\text { Priestley } \\
\text { Cavendish }\end{array}$ & & & \\
\hline & Coulomb & & & \\
\hline & Faraday & & & \\
\hline & Stoney & & & \\
\hline & Crrokes & & & \\
\hline & Hertz & & & \\
\hline & Lenard & & & \\
\hline & J. J. Thompson & & & \\
\hline & Millikan & & & \\
\hline & Röntgen & & & \\
\hline
\end{tabular}

(*) Una contribución o aporte es un hallazgo que no necesariamente derivó en una ley o en un descubrimiento importante, pero del cual se valieron los científicos posteriores para sus trabajos.

(aporte - experiencia - descubrimiento)

2 - ¿Qué importancia tuvieron los hallazgos de Newton para el trabajo posterior de Coulomb? (ley científica) 
3 - Sinteticen los hallazgos de Crookes. ¿¿Sobre qué conocimientos previos se basó? (idem actividad 1)

4 - ¿Cuáles fueron las hipótesis rivales respecto de la naturaleza de los rayos catódicos? ¿Cuál de ellas resulta más creíble? Presenten argumentos a favor de una y de la otra. (hipótesis- hipótesis rivales deducción)

5 - ¿Cuál fue la importancia de las experiencias de Pierre y Marie Curie en relación a la radioactividad para el desarrollo posterior de la Física? (aporte)

6 - Describan el modelo de J. J. Thompson. Formulen dos hipótesis que puedan derivarse de la aceptación de ese modelo. (modelo - hipótesis)

7 - Estudiaremos en detalle la experiencia de Rutherford: (metodología del trabajo científico, etapas)

a) ¿Cuál fue la hipótesis de partida de Rutherford?

b) Identifiquen los conocimientos previos de los que Rutherford se valió para formular su hipótesis. (influencia de los conocimientos previos en la formulación de una hipótesis)

c) Describan el proceso: elaboración de hipótesis - diseño de experimentos - realización de experimentos - interpretación de los resultados - formulación de conclusiones, para el ejemplo en cuestión.

d) ¿Cómo resultó la hipótesis original acerca del átomo, confirmada o refutada? (confirmación - refutación)

e) ¿Piensan que bastó un experimento para echar en tierra la hipótesis? (refutacionismo ingenuo)

f) Este proceso, ¿se vio afectado por el azar? ¿Y por factores extracientíficos? (creencias, actitudes, etc) Justifiquen la respuesta. (influencia de factores extracientíficos)

8 - Con posterioridad a este proceso, se fueron realizando, en forma independiente, una serie de experimentos que condujeron a hallazgos, algunos importantes para la elaboración de otro modelo atómico y los otros, para ir completando el cuadro de la Física Atómica, iniciada antes:

- Realicen una tabla similar a la de la actividad 1, introduciendo algunos de estos nombres (los que ustedes consideren más relevantes): Moseley, Prout, Rutherford, Chadwick, Mc Gill y Soddy, J. J. Thompson y F. W. Aston, Planck, Hertz, Einstein, Millikan, Boltzmann.

- ¿Cómo se vinculan los hallazgos de estos científicos con los de Dalton y Avogadro años antes?

(ciencia como cuerpo de conocimientos en evolución - discusión diacronismo - anacronismo)

9 - ¿Qué hallazgos retoma Bohr para elaborar su modelo? ¿Qué otros tantos permite explicar su nuevo modelo? Elaboren una síntesis sobre este tema. (modelo - explicación - predicción)

10 - Elaboren un mapa conceptual sobre el tema en estudio, de acuerdo con lo que ustedes pudieron comprender sobre la evolución de la Física Atómica. 